\title{
Patrones de compromiso heteroglósico en el marco teórico de artículos científicos en el área de lenguaje*
}

\author{
Heteroglossic engagement patterns in the theoretical \\ framework of research articles in the field of language
}

Diana Chamorro ${ }^{* *}$, Gillian Moss ${ }^{* * *}$, Norma Barletta ${ }^{* * * *}$

\section{RESUMEN}

Este estudio examina cómo los autores construyen su voz autoral. Para ello recurre a las categorías de análisis del sistema de la valoración y su subsistema de compromiso de la lingüística sistémico-funcional. El corpus lo constituye la sección de marco teórico de 20 artículos de investigación en el área de lingüística aplicada en Colombia. El análisis se centra en las cláusulas verbales para describir cómo la voz autoral se ubica en una posición de apertura hacia otras voces (expansión) o, por el contrario, rechaza esas posibles alternativas, o señala un fuerte involucramiento con el punto de vista emitido (contracción). Como resultado, se identificaron tres patrones de compromiso heteroglósico, a saber, diamante, embudo y línea recta. Los patrones identificados son susceptibles de ser enseñados atendiendo a las necesidades y experiencia del escritor, así como a los requerimientos y expectativas de la revista en la que se aspira ser publicado.

\section{ABSTRACT}

This study examines how writers construct their authorial voice using the categories of analysis of the appraisal system and its
Palabras clave:

escritura

académica, compromiso, lingüística sistémicofuncional.
Keywords: academic writing,

Este artículo se inscribe en el proyecto "El uso de los procesos verbales en español para expresar valoración en textos científicos en el área de lenguaje” realizado en la Universidad del Norte, Barranquilla, Colombia.

* Colombiana. Doctora en Investigación, Diagnóstico y Evaluación en la Intervención Educativa. Universidad del Norte, Barranquilla, Colombia. dchamorro@uninorte.edu.co

*** Británica. PhD en Latin American Linguistic Studies. Universidad del Norte, Barranquilla, Colombia.mgilmoss@yahoo.com.ar

***** Colombiana. PhD en Second Language Acquisition and Teaching. Universidad del Norte, Barranquilla, Colombia.nbarlett@uninorte.edu.co 
subsystem of engagement, from a systemic functional linguistic perspective. The corpus is made up of the theoretical framework sections of 20 research articles in the field of applied linguistics in Colombia. The analysis is centered on verbal clauses and describes how the authorial voice acknowledges other voices (expansion), rejects other possible alternatives, or signals a strong involvement with the expressed point of view (contraction). As a result, three patterns of heteroglossic engagement were identified: diamond, funnel and straight line. The patterns identified can be taught based on the needs and experience of the writer, as well as the requirements and expectations of the journal to which the article will be submitted. engagement system, systemic functional linguistics 


\section{Introducción}

La escritura académica es una actividad social con carácter altamente interactivo. Desde Aristóteles se ha distinguido por tener objetivos persuasivos (Hyland, 2008), pues intenta legitimar las aseveraciones, apunta a encontrar un ambiente receptivo entre los lectores e influye en ellos utilizando las convenciones aceptadas, pero también utiliza las voces de los miembros con autoridad dentro de su comunidad disciplinar. Para ello, los escritores dan cuenta de las fuentes, de las posiciones representadas en su texto y negocian con ellas (Hood, 2012); y a menudo intentan anticiparse a posibles reacciones negativas o posiciones alternas utilizando las estrategias, teorías y conocimiento construido previamente (Hyland, 2004).

La escritura de artículos de investigación, en particular, requiere que los investigadores no solo describan los estudios que realizan y sus resultados, sino que lo hagan de tal forma que se evidencie la relación con otros textos y la construcción de la voz autoral. La intertextualidad - que se expresa mediante citas o referencias a otros autores-, constituye muchas veces la base para decidir la publicación de un manuscrito (Hyland, 2004; Myers, 1990). La referencia explícita de los trabajos previos en la sección marco conceptual o teórico forma parte de la construcción colaborativa del nuevo conocimiento y sitúa las aseveraciones del escritor en el marco disciplinario construido hasta el momento. La presencia de otras voces dentro del texto permite establecer un diálogo con las aseveraciones anteriores, con la teoría existente y con los lectores. Esto corresponde a la práctica cada vez más fuerte de contextualizar el trabajo científico en los problemas que se abordan en las disciplinas, así como de estructurar los artículos y también de crear lazos entre la documentación científica (Bazerman, 2000; Hyland, 2004). Se trata, entonces, de crear "una persona profesionalmente aceptable y una actitud apropiada, tanto para los lectores como para la información que se presenta" (Hyland, 2004, p. 13).

La construcción de la voz autoral, identidad de autor o posicionamiento, ha sido estudiada desde diferentes ángulos: a partir de la noción de evidencialidad (Chafe, 1986), el concepto de intensidad (Labov, 1984) y las expresiones de actitudes personales (Biber \& Finegan, 1989). Más recientemente, otros autores se han referido a la voz autoral desde la noción de posicionamiento (stance), relacionado con 
el lenguaje evaluativo, posicionamiento autoral, voz, persona, metadiscurso, atenuaciones (hedging), expresiones enfáticas e identidad de escritor (Hunston \& Thompson, 2000; Pho, 2013).

El posicionamiento y la voz autoral también han sido objeto de estudio dentro de la tradición de la lingüística sistémico-funcional (LSF), desde la teoría de valoración y, más específicamente, a partir de la distinción entre discurso monoglósico y heteroglósico, es decir, un discurso compuesto de una o múltiples voces, respectivamente.

Algunos estudios dentro de esta tradición han estado centrados en la escritura de expertos publicada en revistas académicas y en la escritura de los estudiantes universitarios y preuniversitarios. Al respecto, Chang y Schleppegrell (2011) analizaron la escritura de expertos en revistas en educación y encontraron que con la introducción de varias voces o perspectivas, los escritores crean su nicho, pues exponen las limitaciones de estudios anteriores e introducen explícitamente la brecha entre otras investigaciones y el foco del estudio que presentan.

En cuanto a los estudios referidos a la escritura de estudiantes universitarios y preuniversitarios, el interés se ha centrado especialmente en los ensayos argumentativos. Así, en Italia, Swain (2009) analizó los patrones evaluativos en estudiantes de inglés como segunda lengua; en Qatar, Miller, Mitchell y Pessoa (2014) examinaron ensayos argumentativos universitarios de estudiantes de primer año en el área de historia. Un estudio similar de comparación de ensayos argumentativos fue realizado por Lancaster (2016) en el área de economía y de teoría política en una universidad norteamericana. Así mismo, Aull y Lancaster (2014) compararon el posicionamiento en ensayos argumentativos de entrada a la universidad con los producidos por estudiantes de tercer y cuarto semestre en dos universidades norteamericanas. Además, confrontaron estos datos con artículos de revistas indexadas del Corpus de Inglés Americano Contemporáneo. Por último, en Singapore, Wu (2007) analizó los patrones evaluativos de ensayos de pregrado en geografía escritos por estudiantes con calificación alta y baja.

Los estudios demostraron que los ensayos mejor calificados utilizaban diferentes recursos para admitir otras voces y construir su posi- 
cionamiento a partir del diálogo con ellas, aun cuando los profesores no hubieran hecho explícita esta característica como criterio de evaluación. Los ensayos de bajas calificaciones utilizan más aseveraciones monoglósicas, mientras que los buenos ensayos expresan "distancia crítica" y "alineación discursiva", esto es, incluyen más voces y anticipan las voces o reacciones de los lectores (Lancaster, 2016).

Los resultados de los estudios sugieren que construir una voz autoral de forma adecuada es especialmente difícil para hablantes de segunda lengua; sin embargo, "la tendencia a presentar una persona subjetiva e inapropiada es característico, tanto entre escritores en su primera lengua como en la segunda" (Chang \& Schleppegrell, 2011, p. 41).

En español, en tanto, los estudios se han orientado al análisis de patrones de realización de la voz autoral en documentos históricos (Oteíza, 2010), el discurso pedagógico en las clases de Historia (Manghi \& Badillo, 2015) y las posiciones de los médicos respecto de la práctica (Gallardo \& Ferrari, 2010).

El propósito de la investigación aquí descrita fue analizar, mediante la aplicación de las categorías del sistema de la valoración y el subsistema de compromiso de la LSF, las formas en que los escritores despliegan los recursos léxicogramaticales de las cláusulas verbales en el marco teórico de artículos científicos en español, para posicionarse a sí mismos respecto de otras voces y de las teorías que representan. El análisis de estos significados a nivel semántico-discursivo, a su vez, permite identificar la forma en que su desarrollo construye tres tendencias de patrones reconocibles de compromiso heteroglósico.

\section{Marco referencial}

\subsection{La lingüística sistémico-funcional}

El modelo de la LSF interpreta el lenguaje desde múltiples perspectivas que se complementan para proveer una visión global de los fenómenos lingüísticos. Una de las complementariedades básicas es la de las tres metafunciones, es decir, la idea de que el lenguaje es un recurso para combinar tres tipos diferentes de significado en cada acto de comunicación: significados ideacionales (experienciales y lógicos), textuales e interpersonales. 
Los significados experienciales construyen la experiencia: qué ocurre, quién hace qué y a quién, dónde, cuándo, cómo y por qué. El sistema lingüístico más importante para dar forma a los significados experienciales es la transitividad, que representa lo que existe y ocurre en el mundo, incluyendo el mundo interior. Dicha representación se basa en un principio general para modelar la experiencia: la realidad está conformada por procesos. Según Halliday (2004), la impresión más fuerte de la experiencia es que está compuesta por sucesos de varios tipos: ocurrir, hacer, sentir, significar, ser y devenir. La cláusula, con sus elementos básicos - procesos, participantes y circunstancias-, permite ordenar la variación infinita del flujo de eventos.

Los recursos de la metafunción textual, por su parte, contribuyen al flujo de la información, esto es, cómo los significados experienciales e interpersonales se distribuyen en un texto y cómo se relacionan con otras modalidades de comunicación como la música y las imágenes.

Por último, los significados interpersonales tienen que ver con las relaciones sociales, es decir, con la manera en la que interactúan las personas y qué sentimientos y puntos de vista intentan compartir. Sus sistemas principales son la modalidad, que define el tipo de intercambio (solicitud de información o pregunta, oferta de información o afirmación, solicitud de servicios u oferta de servicios) (Halliday, 2004; Quiroz, 2015, para el caso del español); y la valoración (Hood, 2010; Martin \& White, 2005), que expresa actitudes y grados de compromiso con lo expresado. Los recursos de la valoración se dividen en tres subsistemas: la actitud, que expresa los sentimientos y los juicios del autor o hablante; el compromiso, que tiene que ver con su aceptación o rechazo de las ideas que está comunicando; y la gradación, que indica grados de intensidad de fenómenos, características, actitudes y compromisos.

El estudio aquí presentado abarca aspectos de las metafunciones experiencial e interpersonal. Respecto de la primera, centraremos el análisis en las cláusulas de tipo verbal, es decir, las que tienen que ver con los actos de habla (Halliday, 2004). En cuanto a la metafunción interpersonal, nuestro interés se centra en el sistema de la valoración y, en particular, en el subsistema de compromiso (Martin \& White, 2005), que nos permite describir cómo los escritores construyen su posicionamiento y su relación con referentes propios y de otros. 


\subsection{Sistema de valoración}

El mito de la objetividad del discurso científico fue revaluado hace mucho tiempo (Bazerman, 2000). Sin embargo, a veces persiste la noción de que en los artículos científicos la función persuasiva se limita a las secciones de introducción, discusión y conclusiones. No obstante, estudios minuciosos del discurso científico (Livnat, 2012) han demostrado que la función persuasiva está presente en todas las secciones de los artículos. Esta función es estudiada en la LSF mediante la metafunción interpersonal, específicamente, con el sistema de la valoración y sus subsistemas de actitud y el de compromiso (Martin \& Rose, 2007; Martin \& White, 2005).

Los recursos del sistema de valoración se distribuyen a lo largo de un texto formando patrones prosódicos que dan forma al tono del texto "construyendo el 'posicionamiento' o la 'voz' de quien valora. Este posicionamiento y esta voz definen el tipo de comunidad que se dispone alrededor de valores compartidos" (Martin \& Rose, 2007, p. 59).

La noción de posicionamiento en la LSF está asociada con una "selección de opciones evaluativas para un texto; patrones de uso de opciones evaluativas dentro de un 'tono' (registro) relacionado con un objetivo retórico particular y la construcción de un tipo de persona autoral" (Hood, 2012, p. 55).

La utilización de los recursos del subsistema de compromiso toma características distintivas dependiendo del género y de la disciplina. Así, por ejemplo, en el género de noticias se ha identificado la voz del reportero y la del escritor; asimismo, Coffin (2002), en el discurso de la historia, identifica patrones de recursos que construyen las voces de registrador, interpretador y adjudicador, cada uno con una cantidad y forma particular de expresar opiniones.

El subsistema de compromiso se enmarca en una tradición bajtiniana que presupone que todos los actos comunicativos implican una actitud dialógica en el sentido de que siempre hacen referencia, de alguna forma, a textos anteriores y siempre prevén la reacción de lectores u oyentes reales o potenciales. Esta naturaleza dialógica del discurso puede ser implícita o explícita. Cuando es implícita (el texto no reconoce abiertamente la existencia de otros puntos de vista), decimos que el discurso es monoglósico. Cuando el discurso hace explícita su naturaleza dialógica, decimos que es heteroglósico. En el discurso científico se usan 
tanto la heteroglosia como la monoglosia, según la apertura o no a otras voces y el grado de incertidumbre o certeza que se desee expresar. Por medio del uso de los recursos del compromiso el escritor no solo indica su punto de vista acerca de los contenidos tratados, sino que también busca alinear a sus lectores con su posición.

Por otra parte, los recursos de la valoración se utilizan tanto para establecer una posición en relación con los contenidos presentados por el escritor, como para posicionarse respecto de los contenidos desarrollados por otros investigadores (Hood, 2012). Esta función puede expresarse mediante los procesos verbales cuya elección puede implicar diferentes grados de acuerdo, aprobación, desacuerdo, desaprobación, cuestionamiento, admiración o desprecio, entre otras múltiples opciones (Thompson \& Yiyun, 1991). De este aspecto nos ocupamos en la sección siguiente.

\subsection{Cláusulas verbales}

Las cláusulas verbales desempeñan un papel importante en la construcción de los textos académicos y científicos en los que es necesario hacer referencia a trabajos publicados por otros investigadores (Barletta, Mizuno \& Moss, 2013; Hood, 2010; Ignatieva, 2011; Martin \& White, 2005; Moyano, 2010). Ahora bien, cada comunidad académica disciplinar desarrolla sus propias prácticas para la generación de conocimiento y sistemas de legitimación de dichas prácticas (Maton, 2007). Al respecto, Bazerman (2000) afirma que "cada comunidad, al establecer su propia manera de formular el conocimiento, define qué es el conocimiento en el contexto de la comunidad" (p. 20). De esta manera, los investigadores que aprenden exitosamente a utilizar el discurso de su comunidad para la comunicación de sus ideas -incluyendo el uso apropiado de las cláusulas verbales-, serán aceptados como generadores de conocimiento, mientras que aquellos que no lo logren quedarán marginados.

Desde la perspectiva sistémica, los procesos verbales se sitúan entre los procesos relacionales y mentales, y mediante ellos, se manifiesta la relación simbólica que se construye en la conciencia humana (Halliday, 2014).

En este estudio, decidimos incluir como procesos verbales todos aquellos verbos del decir que proyectan (los tradicionales), por ejemplo, decir, expresar y comunicar, incluyendo aquellos que indican (por 
ejemplo, mostrar y demostrar) (Halliday, 2014); así como también todos aquellos verbos del decir o comunicar que no proyectan (por ejemplo, orar y alabar) y que:

- se consideraban conductuales relacionados con la creación de una representación simbólica (caracterizar, esquematizar, bosquejar, hacer una anotación);

- se encuentran entre lo verbal y material, es decir, los que Matthiessen (1995) llama de "impacto verbal" (acusar, culpar, felicitar, y criticar);

- los procesos que están entre lo verbal y lo que tradicionalmente se ha considerado conductual (hablar, conversar).

Ahora bien, la función de estos procesos verbales varía según el tipo de texto. En los narrativos, por ejemplo, contribuyen a la creación de pasajes dialógicos; en los informes de noticias, permiten al reportero atribuir información a las fuentes, incluidos funcionarios, expertos y testigos oculares (Halliday, 2014); en el discurso académico, permiten no solo citar e informar acerca de estudios realizados, sino que también indican la postura del escritor con verbos como señalar, sugerir, reclamar y afirmar (Halliday 2014). Moyano (2010) identifica, además, las funciones de los procesos verbales en la construcción del campo de la disciplina: referencia a la literatura, confrontación de resultados, realce de los elementos de la argumentación, interpretación de resultados, reconstrucción de los procesos de investigación y la relación entre las partes del artículo.

Así mismo, estudios relacionados con la distribución de los procesos verbales en español demuestran que la frecuencia de su uso varía de acuerdo con el género (Ignatieva \& Rodríguez, 2015). Por su parte, textos de algunos géneros como la revisión pueden incluir un porcentaje significativo de procesos verbales, ya que en estos textos se hace referencia constante a otros autores.

En el caso específico de las revistas especializadas, la investigación de Herrero (2013) también da cuenta de un número significativo de procesos verbales en un corpus de artículos en el área de lingüística aplicada.

\section{Metodología}

El estudio del compromiso heteroglósico en las cláusulas verbales presentado en este artículo se enmarca en la perspectiva sistémicofuncional sobre la valoración (Hood, 2010; Martin \& White, 2005). Los 
resultados aquí presentados hacen parte de la investigación "El uso de los procesos verbales en español para expresar valoración en textos científicos en el área de lenguaje" ${ }^{\prime \prime}$, la cual tiene entre sus objetivos caracterizar los procesos verbales que comportan valoración en el subsistema de compromiso. El análisis en este subsistema permite describir cómo la voz autoral se ubica en una posición de apertura hacia otras voces (expansión); por el contrario, rechaza esas posibles alternativas, o bien, señala un fuerte involucramiento con el punto de vista emitido (contracción).

El corpus está constituido por las secciones de marco teórico de 20 artículos académicos en el área de lenguaje, publicados en cuatro revistas colombianas que se caracterizan por su larga trayectoria, reconocida calidad y su difusión en bases de datos nacionales e internacionales ${ }^{2}$. Hemos analizado la sección de marco teórico porque esta fundamenta y legitima la investigación que se presenta ante la comunidad disciplinar, mediante el reconocimiento de las contribuciones de otros autores al conocimiento acerca del tema objeto de investigación (Hood, 2011). La unidad de análisis es la cláusula verbal, sin embargo, esta no se analiza de manera aislada, sino junto con su cotexto y contexto.

Entre los 20 artículos, 18 desarrollaron la revisión teórica bajo el título explícito de marco teórico, marco referencial, marco conceptual, conceptualización, referente teórico, referencias teóricas, algunas consideraciones previas, o bien, bajo títulos alusivos a los conceptos centrales de la investigación reportada. En un artículo, esta revisión conceptual hizo parte de la sección de introducción y en otro, la reemplazó.

Para realizar el análisis de los recursos de compromiso utilizados en los textos del corpus identificamos, en primer lugar, los procesos verbales; después, los ubicamos en las categorías monoglosia o heteroglosia, esta última con sus respectivas subcategorías (ver Tabla 1). En el proceso de análisis tuvimos en cuenta varias estrategias mediante las cuales la distribución de los procesos verbales y las influencias cotextuales establecieron prosodias de significados interpersonales tales como acoplamiento, propagación, retropropagación (Hood, 2010; Le-

El estudio hace parte del proyecto internacional SAL (Systemics Across Languages). Íkala, Forma y Función, Educación y Educadores, Lenguaje. 
mke, 1988; Martin, 2008) y dominación (Martin \& White, 2005), todo lo cual contribuyó a identificar patrones de compromiso heteroglósico.

Tabla 1.

Sistema de compromiso.

\begin{tabular}{|c|c|c|c|c|}
\hline \multirow{3}{*}{$\begin{array}{l}\text { Contracción } \\
\text { dialógica }\end{array}$} & \multirow{3}{*}{ Rechazo } & \multirow[b]{2}{*}{ Negar } & Absoluto & $\begin{array}{l}\text { Las funciones de } \\
\text { focalización... no habian } \\
\text { sido definidas por los } \\
\text { otros autores }^{3} \text {. }\end{array}$ \\
\hline & & & Atenuado & $\begin{array}{l}\text { Sin embargo, no } \\
\text { podríamos afirmar que } \\
\text { este sería el caso si los } \\
\text { interlocutores estuvieran } \\
\text { frente a frente. }\end{array}$ \\
\hline & & Contradecir & & $\begin{array}{l}\text { El problema no se } \\
\text { encuentra en que el } \\
\text { aprendizaje de un L2 } \\
\text { afecte al L1, sino en lo } \\
\text { que nuestros resultados } \\
\text { indican: que el L1 es } \\
\text { fuente de interferencias } \\
\text { en el bilingüismo... }\end{array}$ \\
\hline \multirow{4}{*}{$\begin{array}{l}\text { Contracción } \\
\text { dialógica }\end{array}$} & \multirow{4}{*}{ Declaración } & Acuerdo & Conceder & $\begin{array}{l}\text { Aunque esta técnica se ha } \\
\text { criticado... }\end{array}$ \\
\hline & & \multirow[t]{2}{*}{ Refuerzo } & Pronunciar & $\begin{array}{l}\text { Es de recalcar; es de } \\
\text { anotar; es importante } \\
\text { señalar; conviene } \text { aclarar; } \\
\text { se puede afirmar; es } \\
\text { necesario referirse a... }\end{array}$ \\
\hline & & & Justificar & $\begin{array}{l}\text { Quizás porque como dice } \\
\text { Cassany; } \\
\text { Searle encontró algunas } \\
\text { fallas... alegando que... }\end{array}$ \\
\hline & & Aprobar & & $\begin{array}{l}\text { Nos guiaremos por la } \\
\text { taxonomía propuesta por } \\
\text { Blum-Kulka, House y } \\
\text { Kasper... }\end{array}$ \\
\hline \multirow{4}{*}{$\begin{array}{l}\text { Expansión } \\
\text { dialógica }\end{array}$} & \multirow[t]{2}{*}{ Consideración } & Alta & & $\begin{array}{l}\text { El papel del lenguaje } \\
\text { en los documentos de } \\
\text { política educativa puede } \\
\text { decirse, entonces, que no } \\
\text { solo es usado por... }\end{array}$ \\
\hline & & Mediana & & $\begin{array}{l}\text { Algunos promueven estas } \\
\text { ideas... }\end{array}$ \\
\hline & \multirow[b]{2}{*}{ Atribución } & Reconocimiento & & Este autor afirma; ... \\
\hline & & Distancia & & $\begin{array}{l}\text { En el aspecto } \\
\text { sintagmático se ha } \\
\text { pretendido }\end{array}$ \\
\hline
\end{tabular}

Fuente: Elaboración propia sobre la base de Martin y White (2005).

$3 \quad$ Los ejemplos son tomados del corpus. 
El acoplamiento se refiere a la forma en que los significados se combinan a través de diferentes metafunciones, estratos o redes de sistemas (Hao \& Humphrey, 2009; Martin, 2008). Por su parte, la propagación es un mecanismo de irradiación de los significados interpersonales mediante conexiones gramaticales o léxicas, incluyendo el acoplamiento, a lo largo de cláusulas y fases más extensas de texto (Hood, 2006). En la retropropagación, los significados se irradian retrospectivamente desde un hiperNuevo (Martin \& Rose, 2007) hacia lo expresado en fases anteriores del texto. En cuanto a la dominación, esta alude al mecanismo por el cual los significados interpersonales de un lugar prominente del discurso, como de un macroTema o un hiperTema, se propagan a las siguientes fases del discurso (Martin \& White, 2005).

Para asegurar la confiabilidad de los análisis, cada investigador analizaba cinco textos, luego los compartía con otro investigador y el análisis acordado entre ambos era socializado con el resto del grupo (seis analistas). Para facilitar y sistematizar el análisis cuantitativo, empleamos el programa Corpus Tool (O’Donnell, 2008) que permitió, una vez realizado el análisis manual cláusula por cláusula, apreciar la distribución en los textos de las diversas categorías de heteroglosia, lo que dio paso a la identificación de patrones.

\section{Resultados}

El análisis de los procesos verbales en los veinte artículos nos permitió identificar tres tendencias que sugieren patrones de realización y despliegue de compromiso heteroglósico que utilizan algunos autores: diamante (cuatro casos), embudo (tres casos) y línea recta (cinco casos). En los demás artículos se apreció un vaivén aparentemente aleatorio entre contracción y expansión, lo que dificulta al lector la identificación del punto de vista del autor ${ }^{4}$. Veamos cada uno de los tres patrones identificados.

\subsection{Patrón diamante}

Consiste en un momento inicial contractivo, seguido de una secuencia expansiva para finalmente cerrar en una nueva contracción.

Ver Hood (2010) acerca de la importancia de la armonía actitudinal. 
En el primer momento contractivo el escritor ${ }^{5}$ elabora una voz autoral revestida de autoridad; para ello, construye un compromiso heteroglósico en el que hace una intervención autoral explícita que enfatiza una postura o afirmación mediante [Heteroglosia: contracción: declaración: refuerzo: pronunciar].

Este compromiso contractivo se expresa frecuentemente mediante el uso de una metáfora interpersonal que antecede al proceso verbal: es importante hacer notar, es de recalcar que. La contracción inicial también puede realizarse en un diálogo heteroglósico con otros autores citados en el que el escritor puede aprobar y conceder: demostraron que..., aunque admiten que..., destacan que...

En un segundo momento, en una secuencia expansiva el escritor abre el espacio para reconocer la posibilidad de otras voces complementarias, diferentes u opuestas respecto de los conocimientos que se revisan. Para ello, puede emplear construcciones verbales como podría afirmarse que..., algunos promueven..., puede decirse..., entonces..., las cuales son típicamente expansivas del tipo [Heteroglosia: expansión: atribución: reconocimiento].

En un tercer momento, este patrón cierra con una nueva contracción en la que el escritor define la teoría o los autores con los que fundamenta su estudio: pero la propuesta que elabora el profesor Montes (1983) resulta pertinente, generalmente del tipo [Heteroglosia: contracción: declaración: aprobar]. do, mientras que autor/a corresponde a las voces citadas. 
Ejemplo 1. Patrón diamante

Además, si se acepta que el aprendizaje de la lengua escrita está basado en la adquisición de la primera lengua, es importante señalar que la mayoría de los niños que nacen sordos no adquieren esta última en el período crítico y que su acercamiento al conocimiento del mundo se hace a través de la experiencia sensorial disponible, y no por una experiencia revestida de lenguaje oral, de un código lingüístico socialmente compartido (Gutiérrez, 2004). (p.35.1)

\begin{tabular}{|c|c|}
\hline $\begin{array}{l}\text { Por otra parte, algunos autores han reconocide que para } \\
\text { escribir exitosamente se requieren habilidades metalin- } \\
\text { guisticas. (p. } 37.3 \text { ) }\end{array}$ & $\begin{array}{l}\text { [Heteroglosia: expan- } \\
\text { sión: atribución: reco- } \\
\text { nocimiento] } \\
\text { Referencia a otros auto- } \\
\text { res }\end{array}$ \\
\hline $\begin{array}{l}\text { Estos procesos psicológicos que menciona Piaget } \\
\text { (1975) son la toma de conciencia, la abstracción y la } \\
\text { autorregulación. ( } \mathrm{p} .38 .3)\end{array}$ & \multirow[t]{2}{*}{$\begin{array}{l}\text { [Heteroglosia: expan- } \\
\text { sión: atribución: reco- } \\
\text { nocimiento] }\end{array}$} \\
\hline $\begin{array}{l}\text { En el mismo sentido, Vygotski (1979) habla de la regulación } \\
\text { ejercida por los otros y la autorregulación. (p. 38.3) }\end{array}$ & \\
\hline $\begin{array}{l}\text { Karmiloff-Smith (1994), desae una perspectiva del } \\
\text { desarrollo, propone un modelo de cambio evolutivo, } \\
\text { basado en la idea de una constante redescripción } \\
\text { representacional de los conocimientos a lo largo del } \\
\text { proceso de aprendizaje (p.39.1) }\end{array}$ & $\begin{array}{l}\text { [Heteroglosia: contrac- } \\
\text { ción: declaración: apro- } \\
\text { bar] } \\
\text { Referencia de anclaje }\end{array}$ \\
\hline
\end{tabular}

Fuente: Elaboración propia.

En el Ejemplo 1, las escritoras recurren a la voz de autoridad de Gutiérrez para construir una postura contractiva mediante es importante señalar, que a su vez se constituye en el punto de partida para el desarrollo de su sustento teórico.

En un segundo momento, las escritoras expanden el horizonte reconociendo a los autores Piaget y Vygotsky, como aportantes al campo que se desarrolla en el artículo, aunque no son clave en la investigación que se presenta; luego, especifican que fundamentarán su marco teórico en los postulados de Karmiloff-Smith. Para esto último utilizan el proceso verbal propone, que adquiere su carácter contractivo [Heteroglosia: contracción: declaración: aprobar] debido a un cotexto constituido por una extensa nota que amplía la propuesta del autor en mención y al contexto, ya que el autor que se menciona (KarmiloffSmith) se retoma varias veces en el siguiente párrafo, en la sección de metodología y en la pregunta de investigación que se formula un poco más adelante en el texto. 


\subsection{Patrón embudo}

Consiste en un momento inicial expansivo seguido de una secuencia contractiva. En el Ejemplo 2, la escritora inicia con una secuencia heteroglósica, dialógica y expansiva, en la que reconoce algunas teorías y postulados existentes en el tema a tratar. Para ello, recurre a procesos verbales como: "dentro de las teorías pedagógicas se han postulado[...] Cesar Coll (Coll et al., 2001) trata de contextualizar" [Heteroglosia: expansión: atribución]. Luego, la escritora inicia la secuencia contractiva que aprueba y delimita el anclaje que sirve de sustento a su investigación: "Este autor [Rico, 2005] afirma que la pedagogía crítica no propone una investigación acerca de la educación sino en y para la educación".

\section{Ejemplo 2. Patrón embudo}

Dentro de las teorías pedagógicas se han postulado diversidad de estrategias y dinámicas docentes, que giran en torno a la relevancia que se le da a un aspecto determinado del proceso de enseñanza-aprendizaje.

Su representante, César Coll (Coll, y otros, 2001), trata de contextualizar la relación psicología-pedagogía, refiriéndose a los apoyos que esta reclama de la primera y delimitando lo que en realidad la psicología puede ofrecer a la pedagogía. (...)

Este autor [Rico, 2005] afirma que "la pedagogía crítica no propone una investigación acerca de la educación sino en y para la educación".

Habermas (citado por Rico, 2005) propone que "los conocimientos sobre investigación generados por una ciencia social crítica, no impulsan necesaria y automáticamente a la acción, sino que es indispensable un proceso de ilustración de los participantes, con el fin de que entiendan una situación determinada y sean capaces de tomar decisiones al respecto".

Es importante mencionar también el tema del aprendizaje significativo, trabajado por John Elliot (citado por Martínez, 2005), que se caracteriza por evitar el malgaste del tiempo del estudiante en la realización de actividades y en aprender contenidos innecesarios o sin sentido [...]

Cuervo y Flórez (1998) mencionan algunos supuestos necesarios para realizar escritos de calidad: [...]

Al revisar los fundamentos de algunas teorías psicológicas en este documento, no se pretende profundizar en cada una de ellas, sino, más bien, mencionar algunos referentes que fundamentan el proceso pedagógico diseñado.

Lev Vigotsky (2000), además del componente biológico madurativo planteado por Piaget en el desarrollo cognitivo, enfatiza el papel de la interacción social, que da origen a los procesos psicológicos superiores, formados por medio de los procesos educativos.
[Heteroglosia:expansión]

Referencia a otros autores

[Heteroglosia:expansión: atribución: reconocimiento] Referencia a otros autores

[Heteroglosia:expansión: atribución:reconocimiento] Referencia clave

[Heteroglosia:expansión: atribución:reconocimiento]

Referencia clave

[Heteroglosia:contracción: declaración:refuerzo: pronunciar]

Metáfora interpersonal

Referencia clave para sustentar la postura [Heteroglosia:contracción: declaración:aprobar]

Heteroglosia:contracción: rechazo:negar:absoluto heteroglosia:contracción: rechazo:contradecir]

Referencia clave para sustentar la postura [Heteroglosia:contracción: declaración:aprobar] 
Allí desempeña un papel importante el lenguaje. Vigotsky (citado por Martínez, 2005) dice que "el éxito del aprendizaje (dentro de un contexto social) está dado por la orientación de las personas adultas (...) de aquí surge la zona de desarrollo próximo [ZDP] [...]".

Ausubel (citado por Martínez, 2005) retoma la teoría del aprendizaje significativo, y afirma que para que este se dé se necesitan tres condiciones: [...]
Referencia clave para sustentar la postura [Heteroglosia:contracción: declaración:aprobar]

Referencia clave para sustentar la postura [Heteroglosia:contracción: declaración:aprobar]

Fuente: Elaboración propia.

En este caso, el proceso verbal afirma es contractivo, porque la escritora se alinea con el enunciado negativo que le sigue y que contradice una postura contraria [Heteroglosia: contracción: rechazo: negar: contradecir]. Esta secuencia incluye una metáfora interpersonal seguida de un proceso verbal: es importante mencionar [Heteroglosia: contracción: declaración: refuerzo: pronunciar] y la aprobación de los aportes de otras voces: "Cuervo y Flórez mencionan [...]. Lev Vigotsky [...] enfatiza [...]. Vigotsky dice [...]. Ausubel retoma [...] y afirma [...]".

El compromiso contractivo se vuelve evidente en el hiperTema: "Al revisar los fundamentos de algunas teorías psicológicas en este documento, no se pretende profundizar en cada una de ellas, sino más bien, mencionar algunos referentes que fundamentan el proceso pedagógico diseñado". Así, la escritora deja claro al lector su compromiso contractivo con los autores que se mencionarán a renglón seguido, aprobando su validez para el estudio que presenta.

\subsection{Patrón línea recta}

La escritora inicia y mantiene una postura heteroglósica contractiva y declarativa. En esta secuencia, la escritora aprueba y establece su acuerdo con los aportes teóricos de autores cuyas contribuciones llegan a constituirse en referencias de anclaje y clave para su trabajo: "En el ámbito latinoamericano, se destacan las investigaciones de Valarino (1994, 1997) y de Carlino (2003d, 2003e). En estos trabajos se señalan [...]. Para efectos de esta investigación es importante destacar el trabajo de Elizabeth Valarino [...]. De acuerdo con Valarino (1997, pp. 231) [...]. El trabajo de Valarino da cuenta [...]. Carlino destaca. [...]”. El patrón se aprecia en el Ejemplo 3. 
Ejemplo 3. Patrón línea recta

En el ámbito latinoamericano, se destacan las investigaciones de Valarino $(1994,1997)$ y de Carlino (2003d, 2003e).

En estos trabajos se señalan los factores que dificultan u obstaculizan la elaboración de una tesis, factores de diversa índole, entre los que se encuentran factores académicos y personales.

Para efectos de esta investigación es importante destacar el trabajo de Elizabeth Valarino (1994, 1997), quien identifica el bloqueo para escribir como una de las variables asociadas a la no terminación de la tesis.
El trabajo de Valarino da cuenta de importantes factores asociados con este problema pero los relaciona exclusivamente con el tesista y no con los demás protagonistas implicados en la elaboración de una tesis.
[Heteroglosia: contracción: declaración: aprobar]

Al contrario, Carlino (2008, p. 187) destaca el papel epistémico que tiene la escritura y que la convierte en

[Heteroglosia: contracción: declaración: aprobar]

[Heteroglosia: contracción: declaración: acuerdo: conceder]

\section{[Heteroglosia:} contracción: una herramienta poderosa para aclarar las propias ideas.

Fuente: Elaboración propia.

A través de varias fases del texto, mediante el acoplamiento de significados ideacionales (proyecciones indirectas, circunstancias y procesos verbales) con significados interpersonales (actitud positiva), la autora crea una armonía consistente que se propaga, asegurando el compromiso contractivo. A su vez, la escritora construye una prosodia de dominación al expresar su compromiso contractivo con Valarino y Carlino, así: "se destacan las investigaciones de Valarino y Carlino [...]", "para efectos de esta investigación es importante destacar el trabajo de Elizabeth Valarino". Este compromiso fluye prospectivamente sobre actitudes que de otro modo no se percibirían contractivas: "el trabajo de Valarino da cuenta [...]; Carlino destaca [...]".

\section{Conclusión}

El análisis de los procesos verbales en la sección de marco teórico de los artículos de investigación de revistas en el área de lingüística aplicada, nos permitió confirmar que las selecciones léxicogramaticales dentro de las cláusulas verbales señalan el compromiso que el escritor construye a medida que desarrolla estas secciones. La selección de un compromiso heteroglósico expansivo está asociada con el reconocimiento del aporte teórico de otros autores, aunque estos no constituyan el fundamento conceptual del trabajo a 
presentar. Asimismo, posibilita al escritor ser reconocido dentro de una comunidad académica como alguien que dialoga con los saberes previamente construidos para hacer nuevos aportes a partir de ellos.

Por su parte, la selección del compromiso heteroglósico contractivo evidencia un diálogo más cerrado, con posturas teóricas más afines entre sí, que implican una mayor identificación e involucramiento para validar el marco de referencia asumido.

El estudio realizado posibilitó también identificar tres posibles patrones de realización de compromiso heteroglósico en las secciones de marco teórico de los artículos científicos en el área del lenguaje: diamante, embudo y línea recta. El primero consiste en un movimiento inicial contractivo, seguido de uno expansivo, para finalizar en un tercero de carácter contractivo. Este patrón diamante parece ser el más amigable, ya que facilita al escritor el planteamiento de su punto de vista, reconocer y referirse a otras posturas y, finalmente, centrarse en las contribuciones teóricas que servirán de fundamento a su trabajo. El patrón embudo, por su parte, se caracteriza por un movimiento inicial expansivo breve, seguido de uno más extenso de tipo contractivo. Este modelo posibilita al escritor iniciar con un reconocimiento de una gama de perspectivas del tema a tratar y continuar con la aprobación de aquellas teorías o marcos conceptuales que se constituyen en el eje de su trabajo. Por último, identificamos el patrón de línea recta, que consiste en la presentación de un solo movimiento contractivo extendido que posibilita al escritor presentar consistente y continuamente las referencias clave o de anclaje que sustentan su trabajo. La ausencia de una contextualización teórica más amplia y de un estado del arte en este patrón, de alguna manera, construye una visión sesgada del asunto.

Por otro lado, los resultados del estudio indican que la selección del verbo léxico para realizar el proceso verbal rara vez implica, por sí sola, el compromiso expansivo o contractivo, sino que son el cotexto y los patrones de prosodia textual los que contribuyen a determinarlo.

Los resultados de este estudio pueden ser utilizados en la enseñanza de la escritura académica en el ámbito universitario en los niveles de pregrado y posgrado, en la medida en que se pueden modelar en los estudiantes tres formas diferentes de escribir un marco teórico, 
atendiendo a la experiencia escritora, al dominio del tema y al espacio disponible.

\section{Referencias bibliográficas}

Aull, L. \& Lancaster, Z. (2014). Linguistic markers of stance in early and advanced academic writing: A corpus-based comparison. Written Communication, 31(2), 151-183. https://doi. org/10.1177/0741088314527055

Barletta, N., Mizuno, J., \& Moss, G. (2013). The use of appraisal resources in the construction of second-language teacher-researcher identity. En G. O'Grady, T. Bartlett, \& L. Fontaine (Eds.), Choice in language: Applications in text analysis (pp. 86-108). London: Equinox.

Bazerman, Ch. (2000). Shaping written knowledge: The genre and activity of the experimental article in science. Recuperado de http://wac.colostate.edu/books/bazerman_shaping/

Biber, D. \& Finegan, E. (1989). Styles of stance in English: Lexical and grammatical marking of evidentiality and affect. Text, 9(1), 93-124. https://doi.org/10.1515/text.1.1989.9.1.93

Chafe, W. (1986). Evidentiality in English conversation and academic writing. En W. Chafe \& J. Nichols (Eds.), Evidentiality. The linguistic coding of epistemology (pp. 261-272). Norwood: Ablex.

Chang, P. \& Schleppegrell, M. (2011). Taking an effective authorial stance in academic writing: Making the linguistic resources explicit for L2 writers in the social sciences. Journal of English for Academic Purposes, 10(3), 140-151. https://doi. org/10.1016/j.jeap.2011.05.005

Coffin, C. (2002). Voices of history: Theorizing the personal semantics of historical discourses. Text, 22(4), 503-528. https://doi. org/10.1515/text.2002.020

Gallardo, S. \& Ferrari, L. (2010). How doctors view their health and professional practice: An appraisal analysis of medical discourse. Journal of Pragmatics, 42(12), 3172-3187. https:// doi.org/10.1016/j.pragma.2010.07.008

Halliday, M. A. K. (2004). An introduction to functional grammar (third edition, revised by Christian M. I. M. Matthiessen). London: Hodder Arnold. 
Halliday, M. A. K. (2014). Halliday's introduction to functional grammar (fourth edition, revised by Christian M. I. M. Matthiessen). London: Routledge.

Hao, J. \& Humphrey, S. (2009). The role of 'coupling' in biological experimental reports. Linguistics and the Human Sciences, 5(2), 169-194. https://doi.org/10.1558/lhs.v5i2.169

Herrero, L. (2013). Transitividad y procesos verbales en el lenguaje académico en español: una perspectiva sistémico-funcional. Lenguas en Contexto, 4, 36-44. Recuperado de http://www. facultaddelenguas.com/lencontexto/app/revista/DIGITAL/4/revista-4.pdf

Hood, S. (2006). The persuasive power of prosodies: Radiating values in academic writing. Journal of English for Academic Purposes, 5(1), 37-49. https://doi.org/10.1016/j.jeap.2005.11.001

Hood, S. (2010). Appraising research: Evaluation in academic writing. London: Palgrave Macmillan.

Hood, S. (2011). Writing discipline: Comparing inscriptions of knowledge and knowers in academic writing. En F. Christie \& K. Maton (Eds.), Disciplinarity (pp. 106-128). London: Continuum.

Hood, S. (2012). Voice and stance as appraisal: Persuading and positioning in research writing across intellectual fields. En K. Hyland \& C. Sancho Guinda (Eds.), Stance and voice in academic genres (pp. 51-68). New York: Palgrave Macmillan.

Hunston, S. \& Thompson, G. (2000). Evaluation in text. Authorial stance and the construction of discourse. Oxford: Oxford University Press.

Hyland, K. (2004). Disciplinary discourses. Social interactions in academic writing. Ann Arbor: University of Michigan Press.

Hyland, K. (2008). Persuasion, interaction and the construction of knowledge: Representing self and others in research writing. International Journal of English Studies, 8(2), 1-23. Recuperado de https://revistas.um.es/ijes/article/view/49151

Ignatieva, N. (2011). Procesos verbales en la escritura académica estudiantil en español desde una perspectiva sistémico-funcional. Lenguaje, 39(2), 447-467. Recuperado de http://www. scielo.org.co/scielo.php?script=sci_abstract\&pid=S0120$34792011000200007 \& \operatorname{lng}=$ en $\& n r m=$ iso 
Ignatieva, N. \& Rodríguez-Vergara, D. (2015). Verbal processes in academic language in Spanish: Exploring discourse genres within the systemic functional framework. Functional Linguistics, 2(1), 2-14. https://doi.org/10.1186/s40554-015-0014-9

Labov, W. (1984). Intensity. En D. Schiffrin (Ed.), Meaning, form and use in context: Linguistic applications (pp. 43-70). Washington DC: Georgetown University Press.

Lancaster, Z. (2016). Expressing stance in undergraduate writing: Discipline-specific and general qualities. Journal of English for Academic Purposes, 23, 16-30. https://doi.org/10.1016/j. jeap.2016.05.006

Lemke, J. (1988). Resources for attitudinal meaning: Evaluative orientations in text semantics. Functions of Language, 5(1), 33-56. https://doi.org/10.1075/fol.5.1.03lem

Livnat, Z. (2012). Dialogue, science and academic writing. Philadelphia: John Benjamins Publishing Company.

Manghi, D. \& Badillo, C. (2015). Modos semióticos en el discurso pedagógico de historia: potencial semiótico para la mediación en el aula escolar. Íkala, Revista de Lenguaje y Cultura, 20(2), 157-172. https://doi.org/10.17533/udea.ikala.v20n2a02

Martin, J. (2008). Tenderness: Realisation and instantiation in a Botswanan town. En N. Nørgaard (Ed.), Odense Working Papers in Language and Communication (Special issued of papers from $34^{\text {th }}$ International Systemic Functional Congress), pp. 30-62.

Martin, J. \& Rose, D. (2007). Working with discourse. Meaning beyond the clause (second edition). London: Continuum.

Martin, J. \& White. P. R. R. (2005). The language of evaluation. Appraisal in English. Basingstoke: Palgrave Macmillan.

Matthiessen, C. (1995). Lexicogrammatical cartography. English systems. Tokyo: International Language Sciences Publishers.

Maton, K. (2007). Knowledge-knower structures in intellectual and educational fields. En F. Christie \& J. R. Martin (Eds.), Language, knowledge and pedagogy. Functional linguistic and sociological perspectives (pp. 87-108). London: Continuum.

Miller, R., Mitchell, T., \& Pessoa, S. (2014). Valued voices: Students' use of engagement in argumentative history writing. Linguistics and Education, 28, 107-120. https://doi.org/10.1016/j. linged.2014.10.002 
Moyano, E. (julio, 2010). Exploring verbal processes in discussions of research articles in Spanish. Exploring verbal processes in discussions of research articles in Spanish. C. En Y Fag y C. Wu (Eds.), Challenges to Systemic Functional Linguistics: Challenges to Systemic Functional Linguistics: Theory and Practice. Proceedings of the 36th International Systemic Functional Congress. Beijing, China: 36th ISFC Organizing Committee.

Myers, G. (1990). Writing biology: Texts in the social construction of scientific knowledge. Madison: University of Wisconsin Press.

O'Donnell, M. (2008). Demonstration of the UAM Corpus Tool for text and image annotation. Recuperado de http://www.wagsoft. com/Papers/ODONNELL-UAMCT-ACL-2008.pdf

Oteíza, T. (2010). Patrones valorativos en el discurso oficial de derechos humanos en Chile: dando valor al pasado y construyendo memorias históricas en la sociedad. Discurso y Sociedad, 4(1), 151-183. Recuperado de http://www.dissoc.org/ediciones/v04n01/DS4(1)Oteiza.pdf

Pho, P. (2013). Authorial stance in research articles: Examples from applied linguistics and educational technology. London: Palgrave Macmillan.

Quiroz, B. (2015). La cláusula como movimiento interactivo: una perspectiva semántico-discursiva de la gramática interpersonal del español. DELTA: Documentação de Estudos em Lingüística Teórica e Aplicada, 31(1), 261-301. https://doi. org/10.1590/0102-445023762456121953

Swain, E. (2009). Constructing an effective 'voice' in academic discussion writing: An appraisal theory perspective. En A. McCabe, M. O'Donnell, \& R. Whittaker (Eds.), Advances in language and education (pp. 166-184). London: Continuum.

Thompson, G. \& Yiyun, Y. (1991). Evaluation in the reporting verbs used in academic papers. Applied Linguistics, 12(4), 365-392. https://doi.org/10.1093/applin/12.4.365

Wu, S. (2007). The use of engagement resources in high- and low-rated undergraduate geography essays. Journal of English for Academic Purposes, 6(3), 254-271. https://doi.org/10.1016/j. jeap.2007.09.006 